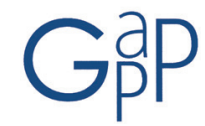

GESTIÓN Y ANÁLISIS DE POLÍTICAS PÚBLICAS, Nueva Época, nº 9 enero-junio 2013 ISSN: 1989-8991

DOI: http://dx.doi.org/10.24965/gapp.voig.10085

\title{
Políticas de regeneración urbana en España: la dimensión sustantiva y procedimental. Del contenido de las políticas a la gobernanza
}

\author{
Rafael Merinero Rodríguez \\ Universidad Pablo de Olavide \\ rmerrod@upo.es \\ María Ángeles Huete García \\ Universidad Pablo de Olavide \\ mahueghar@upo.es \\ Rocío Muñoz Moreno \\ Universidad Pablo de Olavide \\ rmunmor@upo.es \\ Recibido: 2 de octubre de 2012 \\ Aceptado: 30 de julio de 2013
}

\begin{abstract}
Resumen
Desdemediados de la década de los noventa se vienen desarrollando en España actuaciones de regeneración urbana en el marco de las iniciativas impulsadas por la Unión Europea. En este contexto, desde el año 2007, a través de la iniciativa URBANA (2007-2013), se están implementando diversos programas de regeneración urbana para pequeñas y grandes ciudades españolas. Hasta ahora, los trabajos de investigación sobre esta política pública se han centrado en estudios de caso a partir de los elementos fundamentales que inspiran los referidos programas comunitarios, no existiendo, por tanto, ningún trabajo desarrollado en términos extensivos que incorpore un estudio comparado. Este es, precisamente, el objetivo de este trabajo: a partir de un modelo de análisis multidimensional, que persigue recoger los caracteres fundamentales desde la perspectiva del análisis de políticas públicas, realizar un estudio de las políticas locales de regeneración urbana desarrolladas en España en el marco del nuevo programa URBANA (2007-2013), de aplicación de fondos europeos para esta materia.
\end{abstract}

Palabras clave

Nueva gobernanza, área de políticas públicas, regeneración urbana.

\section{Urban Regeneration Policies in Spain. The Substantive and Procedural Dimension. From Policies Content to Governance}

Abstract

From the mid 1990 a number of EU initiatives have enabled the implementation of urban regeneration programmes in Spain. In this context, in the framework of URBANA (2007-2013) several programmes targeted at small and medium sized cities have been implemented since 2007. To date, research on these public policies has focused on the analyses of case studies and, more precisely, on various specific factors common to these European programmes. Therefore no comprehensive analysis carried out from a comparative perspective has been set forth thus far. This is precisely the target of the present work: to conduct the analysis of local policies for urban regeneration carried out in Spain, from the basis of a multidimensional analytical model - which computes the main features with regard to public policies - and within the framework of the new European budget in this field.

Keywords

New governance, public policy area, urban regeneration. 
INTRODUCCIÓN. LAS POLÍTICAS DE REGENERACIÓN URBANA EN ESPAÑA. CARACTERÍSTICAS Y PECULIARIDADES. HACIA UN MODELO BASADO EN LA INTEGRALIDAD

El crecimiento de las ciudades es uno de los hechos más relevantes de las sociedades contemporáneas. Esta circunstancia provoca que los municipios tengan como gran reto afrontar la solución de la mayor parte de los problemas que afectan a las sociedades actuales y atender de manera prioritaria a las necesidades que surgen como consecuencia de esta realidad demográfica y, por extensión, a las necesidades de sostenibilidad urbana que de ello se derivan; sobre todo teniendo en cuenta que las ciudades se han convertido en los motores del crecimiento económico y social a nivel mundial (Florida, 2009). Por lo tanto, uno de los grandes retos de las sociedades contemporáneas, para conseguir la cohesión social, la calidad de vida y la generación de empleo, está en promover el desarrollo urbano.

En este contexto, y desde diferentes esferas institucionales (Comisión Europea, 2000; 2006) y académicas (Sassen, 1999 y 2009; De Gregorio, 2010), se ha venido poniendo de manifiesto que, para cumplir con este reto del desarrollo urbano, una de las líneas instrumentales más importantes la constituyen las intervenciones en barrios y zonas de la ciudad en crisis, ya que las diferencias socioeconómicas, y por tanto los problemas sociales a los que se enfrentan las ciudades, tienen una evidencia socioespacial que se traduce en diferencias significativas entre los barrios.

Dichas iniciativas, se conocen como políticas de regeneración urbana y, de forma general, se definen como aquellas actuaciones destinadas a la recuperación de la actividad económica, la integración social y el medio físico y ambiental de un territorio. De esta forma, la regeneración urbana se refiere a la gestión y planificación de áreas urbanas ya existentes, más que a la planificación y desarrollo de áreas urbanas nuevas (Couch y Fraser, 2003; $\mathrm{Nel} \cdot$ lo, 2010, Roberts y Sykes, 2000, Instituto Universitario de Urbanística de la Universidad de Valladolid, 2010).

Desde finales de los 90, la UE desarrolla una estrategia común para el desarrollo de una política urbana europea', que se pone de manifiesto en varios documentos entre los que destaca, en 2007, la Carta de Leipzig, donde se refleja el concepto de "desarrollo urbano integrado", que incorpora las siguientes dimensiones: medioambiental (movilidad, eficiencia energética, gestión de residuos), social (identidad, cohesión, inclusión), económica (atractivo, inversiones, empleo e innovación) y política (servicios, calidad institucional, participación ciudadana, gobernanza y cooperación público privada) (González, 2011). En definitiva, la forma en que se plantea el desarrollo de una política urbana europea, pasa por implementar actuaciones de carácter integral, en su dimensión sustantiva, que incorporen elementos que atiendan a varias áreas de política pública al mismo tiempo². Además, se incorpora en ellas una dimensión procedimental, denominada por ellos como "compromiso estratégico", en relación con directrices relacionadas con la forma en que estas políticas se gestionan en el interior de la administración. De forma concreta, por una parte, por la propia naturaleza de las iniciativas, su carácter integrado, se establece la necesidad de llegar a acuerdos con los actores implicados en las iniciativas. Por otra parte, se considera necesario incorporar en su gestión mecanismos de eficacia y eficiencia.

En definitiva, desde la importancia que adquieren las políticas de regeneración urbana en las agendas de desarrollo de las ciudades, es necesario poner de manifiesto que las principales características que aquellas adoptan tienen que ver con un modelo de desarrollo urbano integral en el que se sustentan, caracterizado por tres principios fundamentales (Zamora y Merinero, 2012; de Gregorio, 2010):

- El enfoque integrado del desarrollo urbano tiene que ver con tratar la definición y la solución de los problemas teniendo en cuenta de forma conjunta sus dimensiones económicas, culturales, sociales, medioambientales y físicas. Ello supone disponer, en primer lugar, de una visión de conjunto de las cuestiones urbanas sobre las que se pretende intervenir; y después, por la adopción de una perspectiva integral para adoptar las soluciones.

- La articulación de redes de actores e intragubernamental. Así, para la delimitación y caracterización de los problemas, la elaboración de programas y la implementación de las actuaciones no se trata de otra cosa que la ya citada gobernanza territorial³ ${ }^{3}$ es decir, la capacidad de definir e implementar las actuaciones a partir de la colaboración y la cooperación de los diferentes actores públicos y privados que pueden sentirse afectados por los problemas o por las previsibles soluciones. Esta gestión de redes de actores implica la denominada coordinación horizontal (o de los diferentes departamentos de un gobierno), la cooperación vertical (o multinivel entre diferentes tipos

1 Sobre el desarrollo de una política urbana común (Acquis Urbain) y su influencia en la forma que adoptan las políticas públicas de regeneración puede consultarse Atkinson y Rossignolo (2009).

2 Tal como señala un documento del Consejo de Europa (2009): “Urban policy affects the community as a whole and individuals in many aspects of their private lifes. It is a major public act of intervention and direction and therefore be the constant, regulary renewed cooperation between elected representatives and the public. It is part of democratic institutional heritage of tows and cities".

3 A este respecto, puede consultarse Davoudi, Evans, Governa y Santangelo (2008), donde, a partir de la literatura al respecto, se realiza una definición operativa del concepto, en el que se incluyen las dimensiones de la gobernanza consideradas para este artículo. 
de gobierno) y la colaboración de los actores públicos con los privados a través de la cooperación con agentes de la sociedad civil como son empresas o grupos organizados sin ánimo de lucro y la participación ciudadana. Pero además, se hace necesario la aplicación de fórmula de nueva gestión pública que garantice la correcta implicación e incorporación de las diferentes áreas del gobierno que impulsan los programas de regeneración urbana.

- Y el equilibrio intraurbano, es decir, la importancia que cobra el hecho de que los diferentes barios y zonas de la ciudad no presenten estándares de desarrollo socioeconómico y calidad de vida muy diferenciados, pues estas diferencias suelen traducirse en desequilibrios encadenados que pueden llevar a la desestabilización general de la vida en las ciudades. Para que el desarrollo urbano sea equilibrado y beneficie a la ciudad en su conjunto es pues necesario prestar especial atención a estos barrios degradados con el objetivo de reducir las desigualdades e impulsar procesos de cohesión socio-espacial (Borja, 1998; 2003).

Parece evidente pues, que la nueva forma de abordar la intervención en el espacio urbano no se limite a la transformación del espacio físico de la misma, sino que en ello se incluya lo que podríamos denominar, una dimensión social, económica y cultural (Bianchini, 1993). Por ello, en la búsqueda de progreso económico en las ciudades, los proyectos de regeneración urbana tienen como objetivo la búsqueda de una mayor calidad de vida para los individuos de las zonas que los habitan. Ahora bien, la nueva forma de abordar los problemas de los barrios degradados o en crisis no sólo tiene que ver con el contenido de las actuaciones, sino también con la forma en que dichas iniciativas se gestionan, especialmente en relación con el establecimiento de redes de colaboración entre actores públicos y sociedad civil o, dicho de otra forma, el desarrollo de mecanismos de nueva gobernanza local. Es por ello que, el estudio de dichos mecanismos tome especial relevancia en el contexto del análisis de este tipo de políticas, tal como apuntan aportaciones como la realizada por Davies (2002) para el caso de Reino Unido, Rhodes (1997), quien incluso afirma que una de las características definitorias de las políticas de regeneración urbana es la existencia de una red de interdependencia entre los actores públicos y privados; o Paddison (1993), quien apunta la necesidad de superar el concepto de "city marketing" para llegar al de desarrollo urbano integral, pues en ellas se dan dos características: por un lado, la forma de promocionar la política y, por otro, el tipo de alianzas que se forman con los actores que la pueden garantizar.

Hasta el momento, el análisis de las iniciativas de regeneración urbana, tanto en España como en otros países, se ha centrado fundamentalmente en el análisis de su dimensión sustantiva, esto es, el contenido de las iniciativas en atención al tipo de objetivos que se promueven en el municipio (desarrollo económico, mejora de las infraestructuras o fomento de la integración social de sus ciudadanos). Además, dichos análisis se han destinado fundamentalmente al análisis de casos específicos, es decir, al análisis de iniciativas de regeneración adscritas a ciudades o zonas específicas de ciudades concretas.

Sin embargo, son pocos los análisis destinados al estudio de lo que podríamos denominar la dimensión procedimental de estas iniciativas, o dicho de otra forma, la manera en que éstas se gestionan desde las administraciones locales ${ }^{4}$. Menos aun han sido los análisis destinados al estudio de ambas dimensiones y, aún más escasos los intentos de analizar de forma extensiva las iniciativas de regeneración urbana más allá de estudios de casos concretos.

Este trabajo aborda el análisis de ambas dimensiones, tomando como referencia las iniciativas de regeneración urbana desarrolladas en España en el marco de la iniciativa europea URBANA (2007-2013) y tiene la finalidad de aportar un modelo de análisis multidimensional para dar cuenta de la nueva complejidad que caracteriza a las políticas públicas de regeneración urbana. Además, por primera vez, se hace un análisis extensivo de casos, ya que se aborda el estudio de las políticas de regeneración urbana en un número importante de ciudades españolas. Tal como se ha señalado, dicho modelo de análisis se aplica a las ciudades españolas que han puesto en marcha proyectos de regeneración urbana en el marco de la convocatoria URBANA y de Proyectos integrales de desarrollo local y urbano, realizada por los Ministerios de Economía y de Política Territorial del Gobierno de España, en aplicación de medidas a financiar con los Fondos Estructurales para el marco 2007-20135. Esta iniciativa surge con el fin

4 A este respecto destaca el trabajo de González (2011), en el que se analiza de forma comparada para dos ciudades (Santiago de Compostela y Konstanz) el desarrollo de dos dimensiones: el contenido de las políticas y el proceso de las políticas. O bien, el trabajo realizado por Blanco, Bonet y Wallicer (2011) para las ciudades de Madrid y Barcelona.

5 Reglamento (CE) n. ${ }^{\circ} 1083 / 2006$ del Consejo, de 11 de julio de 2006, por el que se establecen las disposiciones generales relativas al Fondo Europeo de Desarrollo Regional, al Fondo Social Europeo y al Fondo de Cohesión y se deroga el Reglamento (CE) n. ${ }^{1260 / 1999,}$

Reglamento (CE) n. $1080 / 2006$ del Parlamento Europeo y del Consejo, de 5 de julio de 2006, relativo al Fondo Europeo de Desarrollo Regional (FEDER), dispone que este Fondo estará destinado a contribuir a la corrección de los principales desequilibrios regionales dentro de la Comunidad, con la finalidad de reducir las disparidades en cuanto al nivel de desarrollo de las distintas regiones y el retraso de las regiones menos favorecidas,

Reglamento (CE) n 1081/2006 del Parlamento Europeo y del Consejo de 5 de Julio, del Fondo Social Europeo (FSE).

Reglamento (CE) n 1084/2006 del Consejo de 11 de Julio, del Fondo de Cohesión (medioambiente e infraestructuras de transporte).

Reglamento (CE) n 1082/2006 del Parlamento Europeo y del Consejo de 5 de Julio sobre la agrupación Europea de Cooperación Transfronteriza (AECT)

Reglamento (CE) n. ${ }^{\circ} 1828 / 2006$ de la Comisión, de 8 de diciembre de 2006, y sus posteriores modificaciones que fijan las normas de desarrollo del Reglamento (CE) n. ${ }^{\circ}$ 1083/2006 del Consejo, así como del Reglamento (CE) n. ${ }^{\circ}$ 1080/2006 anteriormente mencionado, establece la posibilidad de que un 
de dar continuidad a la experiencia obtenida por el desarrollo de la Iniciativa Comunitaria URBAN y los Proyectos Piloto Urbanos desde el año 1994. El objetivo es la puesta en marcha de estrategias innovadoras de regeneración urbana que, mediante un enfoque integrado que contemple los aspectos sociales, económicos y medioambientales, favorezcan un desarrollo urbano sostenible de acuerdo a los principios y orientaciones estratégicas de las políticas comunitarias.

En el marco de este trabajo, se analizan iniciativas desarrolladas en un total de 58 ciudades españolas, tomando en consideración tanto los objetivos y contenido de las mismas (lo que hemos denominado su dimensión sustantiva), como diferentes aspectos relacionados con su gestión (lo que hemos denominado la dimensión procedimental).

El artículo se estructura de la siguiente forma. La primera sección se destina a la propuesta de análisis; tanto en relación con cada una de las dimensiones, procedimental y sustantiva de forma separada, como de manera conjunta. A continuación se expone la propuesta de operacionalización de los conceptos analizados, así como las fuentes de información utilizadas. La segunda sección se destina al análisis de los datos en el mismo orden en el que se plantea al marco analítico; en primer lugar, la dimensión procedimental, a continuación la sustantiva para, al final, analizarlas de forma conjunta. La última sección de destina a las conclusiones.

\section{PROPUESTA PARA EL ANÁLISIS DE LAS POLÍTICAS DE REGENERACIÓN URBANA EN ESPAÑA. MARCO ANALÍTICO BÁSICO, OPERACIONALIZACIÓN Y FUENTES DE INFORMACIÓN UTILIZADAS}

Esta sección se destina a proponer un marco de análisis a partir del cual estudiar las iniciativas de regeneración urbana puestas en marcha en España en el marco de la convocatoria URBANA (2007-2013) desde la perspectiva de la integralidad, es decir, tomando en consideración tanto el contenido y objetivo de las mismas, como la forma en que se gestionan.

Atendiendo a las características definitorias de las políticas de regeneración urbana a las que se han hecho referencia en la sección anterior, podemos atender a dos dimensiones fundamentales. Por una parte, lo que podríamos denominar una dimensión sustantiva, relacionada con el contenido de las políticas: su cobertura en términos de áreas de política pública implicadas, el grado de implicación en el territorio, así como los objetivos que se persiguen para el municipio. Por otra parte, una dimensión que podríamos denominar procedimental, relacionada con la forma en que estas iniciativas se gestionan que, para el artículo que nos ocupa, nos centraremos en el establecimiento de mecanismos de colaboración entre actores de la administración local y con actores ajenos a la misma, es decir, la incorporación de mecanismos de nueva gobernanza.

\subsection{La dimensión procedimental. Modelos de gestión de las iniciativas de regeneración urbana. ¿Cómo se gestio- nan las iniciativas de regeneración urbana en España?}

Tal como se ha señalado anteriormente, una de las características definitorias de las políticas de regeneración urbana es la inclusión de mecanismos de nueva gobernanza local (Davies, 2002; Rhodes, 1997; Paddison, 1993). En este sentido, algunos esfuerzos en la literatura, por ejemplo el realizado por Marshall (2004), pone de manifiesto cómo el desarrollo de directrices de la Unión Europea en materia de políticas urbanas, se refleja en la puesta en marcha de mecanismos de nueva gobernanza, no solamente al nivel Estado Nación, sino de los actores e instituciones locales, tanto en sus patrones de funcionamiento, como en su relación entre ellos y niveles superiores de gobierno ${ }^{6}$. Por esta misma lógica, dado que los municipios incluidos en el análisis de este artículo desarrollan iniciativas de regeneración urbana en el marco de financiación procedente de programas URBANA, se considera relevante conocer la medida en que dichas iniciativas incluyen a su vez mecanismos de nueva gobernanza, tal como se prevé en la literatura al respecto.

En este sentido, la nueva gobernanza local, en el marco de este trabajo, se entendería como el establecimiento de nuevas pautas de interacción entre los municipios y su entorno, por las que se concede mayor protagonismo a los actores civiles en los procesos políticos y en la prestación de servicios (Blair y Stoker, 1991; Painter y Goodwin, 1995), lo que denominaremos "gobernanza externa", así como también otras unidades de gobierno, tanto con otras unidades administrativas municipales (denominadas en el contexto de este trabajo como "gobernanza interna”), como con otras unidades de gobierno, dando lugar a lo que se ha denominado “gobernanza multinivel” (Hooghe y Marks, 2001).

Organismo Intermedio realice una o varias de las tareas de una autoridad de gestión o de certificación, debiendo registrarse formalmente por escrito los acuerdos pertinentes, resultando de aplicación a dicho organismo las disposiciones del citado Reglamento relativas a dichas autoridades (art. 12).

6 De forma concreta, Marshall (2004) hace referencia a tres modificaciones en el ámbito local, a saber: modificaciones en las formas de interacción del gobierno local con niveles de gobierno superiores; incorporación a las actuaciones de gran cantidad y diversidad de actores no gubernamentales y; surgimiento de nuevas agencias (destinadas al desarrollo de partenariados) que van más allá de la propia actuación de regeneración y que se mantienen en el paisaje institucional del municipio una vez que termina la actuación. 
1.2. La dimensión sustantiva: Modelos de intervención en el territorio. ¿Qué objetivos y forma de intervención en el territorio tienen las iniciativas de regeneración urbana?

El análisis de la dimensión sustantiva o, dicho de otra forma, del contenido de la iniciativas, trata de analizar las iniciativas de regeneración urbana a partir del análisis de tres subdimensiones concretas que se detallan a continuación. En primer lugar, en referencia al grado de cobertura de las actuaciones, se considera la cobertura física del territorio. A este respecto se atiende a la medida en que las iniciativas se circunscriben a zonas específicas o a la ciudad en su conjunto. Por otra parte, el análisis de la cobertura, atendería a la medida en que las iniciativas contemplan diversidad en el tipo de iniciativas, entendiendo por diversidad a las diferentes áreas de política pública implicadas en ella. Finalmente, el análisis de la dimensión sustantiva, incluye el estudio de las actuaciones atendiendo al tipo de objetivo prioritario perseguido para el municipio, esto es, el desarrollo económico, la mejora de infraestructuras para el municipio, la integración social de sus ciudadanos ${ }^{7}$, o todas ellas en su conjunto, dando lugar a mayor o menor grado de cobertura política de las intervenciones.

\subsection{La dimensión procedimental y la sustantiva: la gobernanza y el modelo de políticas de regeneración urbana}

Si bien tradicionalmente se han analizado de forma separada las dimensiones procedimental y sustantiva de las políticas de regeneración urbana, son ya algunas las aportaciones en la literatura destinadas a analizar diversos modelos de regeneración urbana en función de los mecanismos de gobernanza puestos en marcha en el marco de estas iniciativas. Dichas aportaciones consideran los mecanismos de gobernanza como factores explicativos de la forma que adoptan finalmente las iniciativas de regeneración urbana. A este respecto, Pierre (1999) sostiene que diferentes formas institucionales de gobernanza sustentadas en distintos sistemas de valores, normas, creencias y prácticas producen distintas elecciones de política pública así como sus resultados. De forma concreta, en relación con el análisis de políticas de regeneración urbana, artículos como el realizado por Parés, Bonet-Marti y Martí-Costa (2011) así como el trabajo de Blanco (2009) y Brendan y Shine (2001), encuentran una relación entre los mecanismos de gobernanza y el grado de cobertura política de las iniciativas, afirmando que modelos más participativos tienden a ser a su vez aquellos desarrollados en políticas de regeneración con un carácter más amplio y una mayor orientación hacia el desarrollo social y comunitario del municipio. Por su parte, Jones y Evans (2006), plantean tres modelos de políticas de regeneración asociadas a tres modelos de gobernanza a lo largo del tiempo, encontrando modelos más pluralistas en lo que a la implicación de actores se refiere asociados a políticas más diversas en cuanto al tipo de actuaciones a desarrollar.

Este artículo plantea por el contrario que, en el marco de las propuestas teóricas sobre los dominios de políticas públicas, el carácter de las iniciativas de regeneración, tanto en su cobertura como en la naturaleza de los objetivos perseguidos para el municipio, configura un contexto o estructura de oportunidad que propicia la cantidad y tipo de actores implicados en la misma. En la lógica de las teorías sobre arenas de política local, que si bien tal como señala la propuesta de Lowi (1964) diferentes políticas (policies) dan lugar a diferentes dinámicas políticas (politics), Scharpf (1997) señala que las primeras fijan además qué "constelaciones de actores" intervienen en ellas, esto es, los actores que realmente se encuentran implicados en la interacción en el seno de una política, sus capacidades, percepciones y evaluaciones que éstos hacen sobre los beneficios a obtener de la misma, y la medida en que sus expectativas sobre sus beneficios son compatibles o incompatibles con otros (Scharpf ,1997:44). Así, aquel conjunto de actores y sus pautas específicas de interacción, relacionadas con una policy concreta, conformará lo que suele denominarse una "arena de políticas" o "dominio político"(policy domains) (Burstein, 1991; Knoke, 1990). Ello, en definitiva, conformará una institución política específica (policy institution), en el sentido de que cada "arena de políticas" o "dominio político" contiene una serie de reglas y normas, formales o informales, que determinan criterios de inclusión/exclusión para los actores y el curso de la toma de decisiones en el seno de la misma (Ostrom, 1986; Scharpf, 1997).

En este sentido, en la línea de las propuestas teóricas, se plantea que, en función del grado de cobertura de las actuaciones, así como del tipo de objetivo prioritario perseguido para el municipio, las iniciativas de regeneración urbana constituyen un contexto o estructura de oportunidades propicia para la participación de más o menos actores, así como el carácter de los mismos. De forma concreta, se plantea que actuaciones más orientadas a la búsqueda de desarrollo económico en el municipio, se caracterizarán por el establecimiento de mecanismos de gobernanza con mayor participación de actores económicos del municipio, por ser estos quien con sus intereses y recursos podrían garantizarlos. Por el contrario, actuaciones destinadas a la promoción del bienestar social, contrarían con mayor participación de entidades ciudadanas de carácter cívico, por ser estas las más implicadas en este tipo de objetivos y las que mayores recursos tendrían para la participación en este tipo de políticas. Finalmente, se considera que a medida que las iniciativas alcancen una mayor cobertura política y territorial necesitarán

7 Sobre exclusión social e iniciativas de regeneración urbana, puede consultarse la amplia producción de Atkinson (1999). 
de la colaboración o acuerdos con mayor número y diversidad de actores en el municipio, dando lugar a mayores niveles de nueva gobernanza en el municipio.

A continuación se presentan las dimensiones procedimental y sustantiva, así como la forma en que se han medido de cara a su posterior análisis:

TABLA 1. MOdelo DE POLÍTICA DE REGENERACIÓN URBANA. S PERSIGUEN EL DESARROLLO ECONADRMA EN QUE LAS DIMENSIONES SEEGRACI MEDIDA EN QUE LAS ACTUACIONES PERSIGUEN EL DESARROLLO ECONAD

\begin{tabular}{|c|c|c|c|}
\hline CONCEPTO & DIMENSIÓN & SUBDIMENSIÓN & ÍTEM \\
\hline \multirow{6}{*}{$\begin{array}{l}\text { MODELO POLÍTICA } \\
\text { DE } \\
\text { REGENERACIÓN URBANA }\end{array}$} & \multirow{3}{*}{ PROCEDIMENTAL } & Gobernanza interna & $\begin{array}{l}\text { Áreas municipales con las que se ha } \\
\text { coordinado para la ejecución del proyecto }\end{array}$ \\
\hline & & Gobernanza externa & $\begin{array}{l}\text { Entidades de la sociedad civil con las que se } \\
\text { ha coordinado para la ejecución del proyecto }\end{array}$ \\
\hline & & Gobernanza multinivel & $\begin{array}{l}\text { Niveles de gobierno con los que se ha coordi- } \\
\text { nado para la ejecución del proyecto }\end{array}$ \\
\hline & \multirow{3}{*}{ SUSTANTIVA } & Cobertura territorial & $\begin{array}{l}\text { Beneficio solo para la zona objeto de inter- } \\
\text { vención vs. la ciudad en su conjunto }\end{array}$ \\
\hline & & Cobertura política & $\begin{array}{l}\text { Áreas posibles de intervención/Áreas que se } \\
\text { desarrollan }\end{array}$ \\
\hline & & Objetivos que promueve & $\begin{array}{l}\text { Importancia/prioridad del objetivo según } \\
\text { área de política pública }\end{array}$ \\
\hline
\end{tabular}

Fuente: elaboración propia.

\subsection{Fuentes de información utilizadas}

Para la obtención de la información necesaria para la realización de los análisis, se ha recurrido a fuentes primarias, a través de la administración de un cuestionario entre técnicos municipales implicados en la gestión y ejecución de las iniciativas de regeneración urbana. De dicha herramienta se adjunta ficha técnica a continuación:

TABLA 2. FICHA TÉCNICA DEL CUESTIONARIO

\begin{tabular}{|l|c|c|c|c|} 
& Municipios mayores de 50.000 habitantes & $\begin{array}{c}\text { Municipios entre 20.000 y } \\
50.000 \text { habitantes }\end{array}$ \\
$\mathbf{N}$ & 46 & 34 & 56 \\
$\mathbf{n}$ & $73,9 \%$ & 24 \\
Tasa de respuesta & 0,0868 & $42,9 \%$ \\
Error & $91,32 \%$ & 0,1526 \\
Nivel de confianza & Dic 2010 -nov 2011 & $84,74 \%$ \\
Periodo de desarrollo del trabajo de campo & Dic 2010 -nov 2011
\end{tabular}

Fuente: elaboración propia.

El siguiente apartado se destina a mostrar los resultados obtenidos una vez realizados los análisis pertinentes de las dimensiones descritas anteriormente. Para ello se han utilizado comparaciones de medias, tablas de contingencia, cruces entre variables y análisis factorial.

\section{LAS POLÍTICAS DE REGENERACIÓN URBANA EN ESPAÑA EN EL MARCO DE LA INICIATIVA URBANA 2007- 2013. RESULTADOS EMPÍRICOS}

Esta sección se destina al análisis de la información obtenida de la administración de la encuesta entre los técnicos municipales implicados en la gestión e implementación de iniciativas de regeneración urbana en España, desarrolladas bajo la iniciativa europea URBANA, en la convocatoria 2007-2013. Para ello, en primer lugar se analizan las principales características relacionadas con la forma en que se gestionan los programas, concretamente, en atención al establecimiento de mecanismos de gobernanza. En segundo lugar, se analiza el contenido de las iniciativas, atendiendo de forma concreta a su grado de cobertura política y territorial, así como los objetivos que se promueven para el municipio con la puesta en marcha de estos programas. Finalmente se hace un análisis conjunto de ambas dimensiones.

\subsection{Dimensión procedimental de las iniciativas de regeneración urbana en España}

A continuación se muestran los resultados del análisis de estrategias de nueva gobernanza local, a través del establecimiento de redes de colaboración entre las unidades administrativas del ayuntamiento (gobernanza inter- 
na), con otros niveles de gobierno (gobernanza multinivel) o con actores de la sociedad civil local, como empresas y entidades ciudadanas (gobernanza externa).

\subsubsection{Desarrollo de mecanismos de gobernanza interna}

En relación con la primera de las dimensiones de la nueva gobernanza, en general, el grado de colaboración entre áreas del ayuntamiento dentro de las iniciativas de regeneración es de un nivel que podríamos denominar medio/bajo. Sin embargo, si analizamos dicha colaboración atendiendo a cada una de las áreas del ayuntamiento implicadas, observamos una mayor implicación del área de urbanismo, tal como cabría esperar de este tipo de intervenciones, muy ligadas a transformaciones del carácter físico del municipio. Ello mismo se pone de manifiesto en estudios como el desarrollado por González (2011), quien apunta este aspecto como una característica propia de los municipios españoles, frente a otros casos en Europa. En segundo lugar, lo serían las áreas de economía y medio ambiente, siendo las menos implicadas en los procesos de colaboración áreas como vivienda y deporte.

TABLA 3. COLABORACión ENTRE ÁREAs del AYUNTAMIENTO

\begin{tabular}{|l|r|r|}
\hline \multicolumn{1}{|c}{ Áreas municipales } & Media & Desviación típica \\
\hline Urbanismo & 63,66 & 34,02686 \\
\hline Economía & 47,32 & 37,52933 \\
Medio Ambiente & 47,32 & 37,83263 \\
\hline Empleo & 40,28 & 41,19542 \\
\hline Bienestar Social & 37,46 & 37,78580 \\
\hline Cultura & 25,07 & 35,73207 \\
\hline Turismo & 12,95 & 27,69189 \\
\hline Deporte & 9,57 & 26,58875 \\
\hline Vivienda & 8,73 & 26,34548 \\
\hline Media total & 32,48 & - \\
\hline
\end{tabular}

Fuente: elaboración propia.

Nota: Indicadores medidos en una escala 0_100.

Ahora bien, cabría plantearse si dichas redes de colaboración varían en función del área o delegación municipal responsable principal de las iniciativas, entendiendo, en la lógica de las teorías sobre arenas de política local, que diferentes políticas (policies) darían lugar a diferentes dinámicas políticas (politics).

En este sentido, los análisis han puesto de manifiesto que dichas redes de colaboración apenas varían si consideramos separadamente el área responsable de la intervención. Tal como se pone de manifiesto en la siguiente tabla, las redes de relación con otras áreas municipales se establecen de forma similar, con independencia de quien sea el área responsable de la iniciativa.

TABLA 4. COORDINACIÓN ENTRE ÁREAS ATENDIENDO AL ÁREA RESPONSABLE DE LA INICIATIVA

\begin{tabular}{|c|c|c|}
\hline OFICINA O ÁREA RESPONSABLE & Área con la que existe mayor coordinación & Indicador (media 0_100) \\
\hline \multirow{3}{*}{ Oficina creada expresamente } & Empleo & 61,25 \\
\hline & Urbanismo & 52,50 \\
\hline & Economía & 52,50 \\
\hline \multirow{4}{*}{ Urbanismo } & Economía & 30 \\
\hline & Medio Ambiente & 30 \\
\hline & Empleo & 16,67 \\
\hline & Bienestar Social & 16,67 \\
\hline \multirow{3}{*}{ Economía } & Urbanismo & 55 \\
\hline & Empleo & 55 \\
\hline & Medio Ambiente & 50 \\
\hline \multirow{4}{*}{ Empleo } & Medio Ambiente & 80 \\
\hline & Urbanismo & 65 \\
\hline & Economía & 50 \\
\hline & Bienestar Social & 50 \\
\hline
\end{tabular}




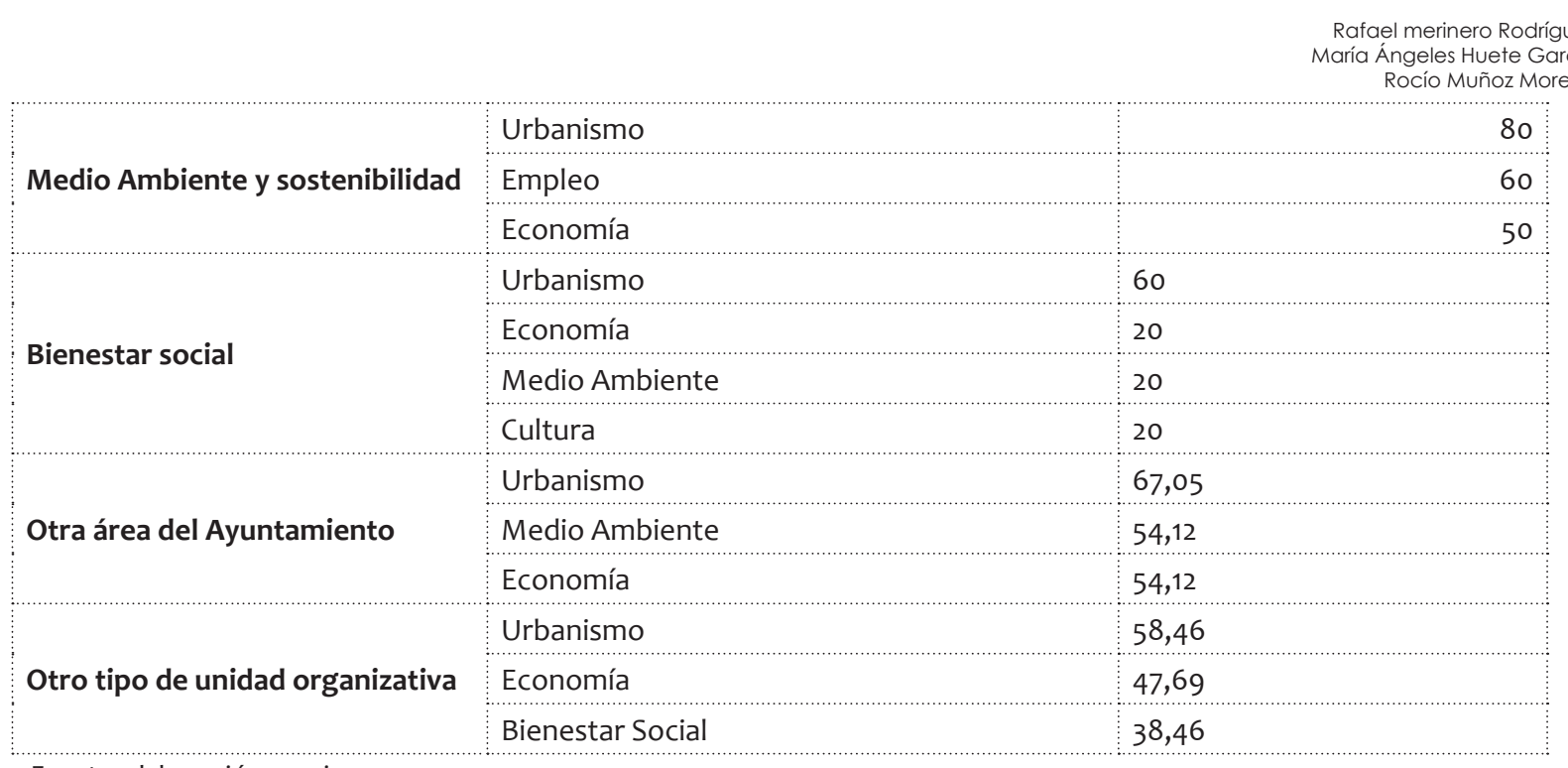

Fuente: elaboración propia.

Nota: Indicadores medidos en una escala 0_100.

\subsubsection{Desarrollo de mecanismos de Gobernanza multinivel}

En atención al establecimiento de mecanismos de gobernanza entre diferentes niveles de gobierno, se pone en evidencia una colaboración especialmente alta con administraciones situadas en niveles superiores, especialmente el nivel autonómico y central y, en menor medida con la Diputación Provincial y otros municipios. Es importante señalar que la baja colaboración entre municipios y Diputación provincial posiblemente se deba al tamaño de los municipios incluidos en la muestra, todos ellos mayores de 20.000 habitantes, los cuales son precisamente los menos proclives a la colaboración con esta unidad de gobierno, tal como se establece en la Ley de Bases de Régimen local que regula las relaciones entre unidades de gobierno en el caso español.

Ahora bien, con independencia de los casos analizados, este resultado se pone de manifiesto en otros estudios, por ejemplo, en el trabajo de Kears y Turok (2000) o Davies (2002) para el caso de Reino Unido se evidencia una relación de superioridad entre el gobierno central y el resto de unidades de gobierno. Tal como señalan estos autores, por una parte, el gobierno central sigue teniendo más poder y lo que hace es redistribuir responsabilidades entre el resto de actores. Por otra parte, desde el punto de vista de los actores locales, el establecimiento de mecanismos de gobernanza se darían como resultado de un principio de competitividad que establecería el gobierno central, al objeto de poder acceder a los recursos con los que desarrollar iniciativas de regeneración urbana. En este sentido, el establecimiento de mecanismos de gobernanza en el seno de las administraciones locales sería más bien una cuestión de forma que un resultado real.

Ello mismo podría estar sucediendo en el caso español, más aún si analizados los patrones de gobernanza entre administraciones en relación a la naturaleza de la misma, fundamentalmente de carácter financiero, por lo que la relación de colaboración se daría más bien a partir de un principio de dependencia financiera.

TABLA 5. COLABORACIÓN CON OTROS NIVELES DE GOBIERNO

\begin{tabular}{|c|c|c|c|c|}
\hline & Mínimo & Máximo & Media & Desviación típica \\
\hline Otros Municipios & , o० & 66,67 & 3,80 & 12,09578 \\
\hline Diputación Provincial &, 00 & 83,33 & 2,85 & 12,99431 \\
\hline Gobierno Autonómico &, 00 & 100,00 & 10,71 & 24,08314 \\
\hline Gobierno Europeo & , o० & 100,00 & 12,85 & 26,49270 \\
\hline Gobierno Central &, 00 & 100,00 & 28,80 & 37,31330 \\
\hline I.S. Gobernanza Multinivel & , o० & 56,67 & 11,80 & 13,28113 \\
\hline
\end{tabular}

Fuente: elaboración propia.

Nota: Indicadores medidos en una escala 0_100.

\subsubsection{Desarrollo de mecanismos de gobernanza externa}

En atención con la colaboración con actores de la sociedad civil local, se ha puesto de manifiesto que los mayores niveles de gobernanza se dan con las entidades ciudadanas y en menor medida con empresas. Estos resultados muestran un carácter cívico de la gobernanza en las políticas de regeneración urbana analizadas, frente a un 
carácter más empresarial, como cabría esperar en el marco de estas iniciativas, dado su carácter tradicionalmente orientado a la búsqueda del desarrollo económico del municipio, de acuerdo con las teorías relacionadas con las coaliciones de gobernanza (Castells, 1986; Logan y Molotch, 1987; Stone, 1989).

TABLA 6. GOBERNANZA EXTERNA. INTENSIDAD

\begin{tabular}{|l|r|r|r|r}
\hline & Mínimo & Máximo & Media & Desviación típica \\
\hline $\begin{array}{l}\text { Entidades con ánimo de lucro (empresas) } \\
\text { Entidades sin ánimo de lucro (asociaciones) }\end{array}$ &, 00 & 100,00 & 14,52 & 26,14856 \\
\hline
\end{tabular}

Fuente: elaboración propia.

Nota: Indicadores medidos en una escala 0_100.

En definitiva, de los análisis de la dimensión procedimental se desprende el establecimiento de mecanismos de coordinación especialmente en el ámbito local, ya sea entre áreas municipales o con actores de la sociedad local, siendo menor entre administraciones, especialmente con otros municipios y Diputación provincial.

TABLA 7. MECANISMOS DE GOBERNANZA. INTENSIDAD: INDICADORES SINTÉTICOS

\begin{tabular}{|l|c|}
\hline \multicolumn{2}{|c|}{ Índices sintéticos de intensidad de gobernanza } \\
\hline Gobernanza interna & 32,48 \\
Gobernanza multinivel & 11,81 \\
Gobernanza externa & 23,21 \\
\hline Gobernanza total & 22,63
\end{tabular}

Fuente: elaboración propia.

Nota: Indicadores medidos en una escala 0_100.

\subsection{Dimensión sustantiva de las iniciativas de regeneración urbana en España}

El análisis del contenido de las iniciativas trata de analizar las actuaciones de regeneración urbana a partir del análisis de tres subdimensiones concretas, como son, cobertura territorial y política y el objetivo perseguido para el municipio.

En atención al grado de cobertura de las actuaciones. Atendiendo a la cobertura física del territorio, los análisis ponen de manifiesto que la mayoría de ellas se circunscriben a un término medio, esto es, a actuaciones que van más allá de una zona específica, pero que no abarcan a la ciudad en su conjunto. A la luz de los resultados, podría afirmarse que las iniciativas de regeneración urbana no se estarían circunscribiendo al conjunto de la ciudad, considerada como un todo, sino que más bien constituirían enfoques fragmentados y parciales, a excepción de una pequeña parte, el 12,89\% del total de la muestra, cuyas actuaciones afectarían a la ciudad en su conjunto.

TABLA 8. COBERTURA TERRITORIAL DE LAS ACTUACIONES

\begin{tabular}{|c|c|}
\hline COBERTURA TERRITORIAL DE LAS ACTUACIONES & PORCENTAJE DE RESPUESTAS \\
\hline Solamente una zona concreta (1) & 10,06 \\
\hline 2 & 5,67 \\
\hline 3 & 4,82 \\
\hline 4 & 5,95 \\
\hline 5 & 21,95 \\
\hline 6 & 23,23 \\
\hline 7 & 3,26 \\
\hline 8 & 3,12 \\
\hline 9 & 9,07 \\
\hline La ciudad en su conjunto (10) & 12,89 \\
\hline Media (0_10) & 5,25 \\
\hline
\end{tabular}

Fuente: elaboración propia.

Nota: Indicadores medidos en una escala 0_10.

En atención a la cobertura política, esto es, la medida en que las iniciativas desarrolladas atienden a varias áreas de política pública, las actuaciones tienen un índice de cobertura política de 31,47, en una escala o_100. Ello 
supone un índice de cobertura política medio- bajo. Ello muestra por tanto que, si bien es un principio planteado en el marco de las iniciativas desarrolladas en el contexto de la iniciativa URBANA (2007-2013), aún no existe una amplia cobertura en cuanto a la diversidad de áreas de política pública implicadas en las mismas.

\section{TABLA 9. COBERTURA POLÍTICA DE LAS ACTUACIONES}

\begin{tabular}{|c|c|c|c|c|c}
\hline & Mínimo & Máximo & Media & Desv. típ. \\
Media cobertura política & 13,39 & 67,86 & 31,02 & 11,55026
\end{tabular}

Fuente: elaboración propia.

Nota: Indicadores medidos en una escala 0_100.

Finalmente, el análisis de la dimensión sustantiva, incluye el estudio de las actuaciones en atención al tipo de objetivo perseguido para el municipio. Para ello se consultó sobre una amplia diversidad de actuaciones que, tras el análisis factorial realizado, se redujeron a los indicadores sintéticos con los que finalmente se trabaja.

Tal como se pone de manifiesto en la siguiente tabla, los municipios que desarrollan iniciativas de regeneración urbana en España, tienen como objetivo principal el incremento de la oferta de equipamientos y servicios en el municipio, seguido de la creación de empleo y nuevos nichos económicos, fomento de la sociedad de la información y, finalmente, el fomento de la cohesión social y la accesibilidad.

\section{TABLA 10. OBJETIVO PERSEGUIDO PARA EL MUNICIPIO}

\begin{tabular}{|c|c|c|c|}
\hline \multicolumn{2}{|l|}{ Objetivo perseguido por la iniciativa } & \multirow{4}{*}{$\begin{array}{r:}\text { Media } \\
57,14\end{array}$} & \multirow{4}{*}{$\begin{array}{r}\text { Alfa de Cronbach } \\
0,083\end{array}$} \\
\hline Fomento de la sociedad de la información y nuevas tecnologías & \multirow{3}{*}{$\begin{array}{l}\text { Fomento de la sociedad de } \\
\text { la información }\end{array}$} & & \\
\hline $\begin{array}{l}\text { Búsqueda de nuevas fuentes de desarrollo económico en } \\
\text { el municipio (nuevas áreas de producción ligadas al } i+d+i)\end{array}$ & & & \\
\hline Mejoras en la movilidad (peatonalización de calles) & & & \\
\hline Creación de empleo en el municipio & \multirow{2}{*}{$\begin{array}{l}\text { Creación de empleo y nue- } \\
\text { vos nichos económicos }\end{array}$} & \multirow[b]{2}{*}{75,71} & \multirow[b]{2}{*}{0,732} \\
\hline $\begin{array}{l}\text { Búsqueda de otras fuentes de desarrollo económico en el } \\
\text { municipio (nuevos nichos económicos) }\end{array}$ & & & \\
\hline \multicolumn{2}{|c|}{ Media agregada de actuaciones destinadas a la promoción económica } & \multicolumn{2}{|l|}{66,42} \\
\hline $\begin{array}{l}\text { Mejoras en la accesibilidad a equipamientos, servicios pú- } \\
\text { bicos o viviendas }\end{array}$ & \multirow{3}{*}{$\begin{array}{l}\text { Cohesión social y accesibi- } \\
\text { lidad }\end{array}$} & \multirow{3}{*}{64,78} & \multirow{3}{*}{0,635} \\
\hline Fomentar la identidad del municipio (sentimiento de pertenencia) & & & \\
\hline Fomentar la identidad del barrio & & & \\
\hline $\begin{array}{l}\text { Aumento de la oferta en equipamientos públicos al servicio } \\
\text { de la ciudadanía }\end{array}$ & \multirow{2}{*}{$\begin{array}{l}\text { Aumento de equipamien- } \\
\text { tos y servicios }\end{array}$} & \multirow{2}{*}{83,57} & \multirow{2}{*}{0,718} \\
\hline $\begin{array}{l}\text { Aumento de la oferta de servicios públicos al servicio de la } \\
\text { ciudadanía }\end{array}$ & & & \\
\hline \multicolumn{2}{|c|}{ Media agregada de actuaciones destinadas a la promoción del bienestar social } & 74,18 & \\
\hline
\end{tabular}

En definitiva, del análisis del tipo de objetivos perseguidos para el municipio, se desprende que, tal como se vienen señalando en la literatura al respecto (Bianchini, 1993; Borja, 1998; Zamora y Merinero, 2012), las iniciativas de regeneración urbana ya no solamente se circunscriben a la búsqueda de la promoción económica del municipio, sino que en ellas se busca además la consecución de otros objetivos, como es la búsqueda de bienestar, bien a través de la mejora de equipamientos y servicios públicos, o bien a través de actuaciones destinadas la integración social de la ciudadanía.

\subsection{Cruce de las dos dimensiones: la dimensión procedimental en función de la dimensión sustantiva}

Tal como se planteaba al inicio, este artículo plantea el análisis de la relación entre la dimensión procedimental y sustantiva o, dicho de otra forma, el desarrollo de mecanismos de gobernanza y su relación con la forma que adoptan las políticas de regeneración urbana. En este sentido, en primer lugar, la dimensión sustantiva se ha analizado en atención a su cobertura, tanto desde el punto de vista territorial (su grado de implicación en el territorio), como desde el punto de vista político (al grado de implicación de áreas de política pública en su desarrollo).

Tal como se pone de manifiesto en los análisis, no existe relación entre la cobertura territorial y el desarrollo de mecanismos de gobernanza, con independencia de con quien se desarrollen. Es decir, el grado de implicación en el territorio, solamente una zona frente a la ciudad en su conjunto, no implicaría el desarrollo de mas o menos mecanismos de colaboración con actores ajenos a la administración local, ya sean de carácter público o privado. 
Ahora bien, tal como se pondría en evidencia tanto en las hipótesis planteadas al inicio de este trabajo, como en la literatura al respecto (Parés, Bonet-Marti y Martí-Costa, 2011; Blanco, 2009, Brendan y Shine, 2001; Jones y Evans, 2006), la existencia de una mayor pluralidad de áreas de política local estaría en relación con una mayor importancia de mecanismos de gobernanza, de forma concreta, con actores de la sociedad local. Es decir, a medida que se implicase un mayor número de áreas de política local para el desarrollo de la iniciativa de regeneración urbana, se requeriría de la implicación y acuerdos con mayor número y diversidad de actores de la sociedad local, así como otros niveles de gobierno, con los que sería necesaria la colaboración para el desarrollo de esta política local. Ello además se pone en evidencia en otros documentos, por ejemplo, del Consejo de Europa (2009), en el cual se afirma que en este tipo de políticas urbanas, dado que afectan a la comunidad en su conjunto, requieren de una constante cooperación entre los actores públicos y la sociedad.

\section{TABLA 11. COBERTURA TERRITORIAL Y POLÍTICA EN FUNCIÓN DEL TIPO DE GOBERNANZA}

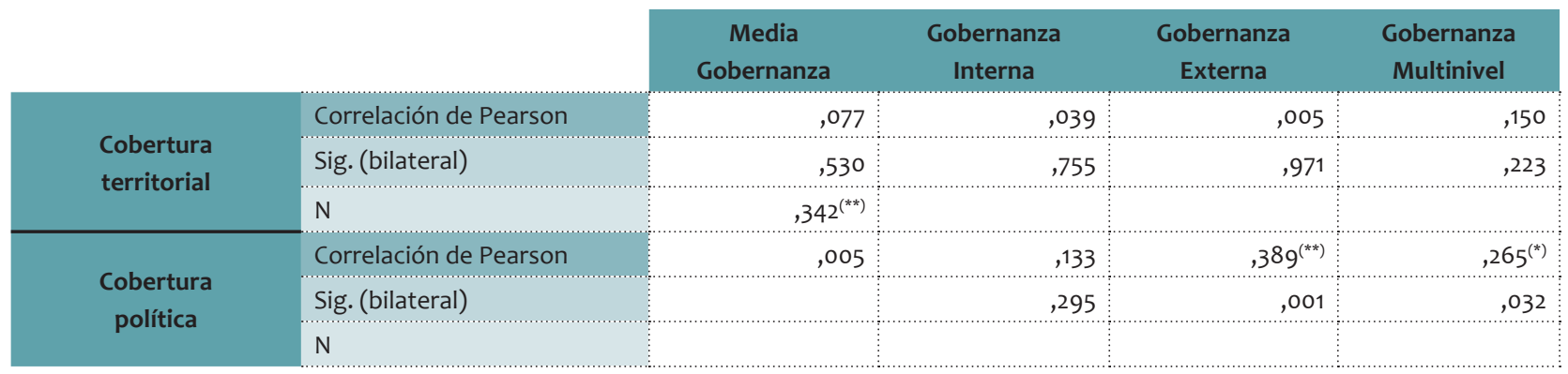

Fuente: elaboración propia.

**. La correlación es significativa al nivel 0,01 (bilateral).

*. La correlación es significativa al nivel 0,05 (bilateral).

Por su parte, en relación con el desarrollo de mecanismos de gobernanza en función del área de política local, analizado a través de los objetivos perseguidos para el municipio con el desarrollo de la iniciativa de regeneración urbana, los análisis ponen de manifiesto que existen diferencias entre áreas de política pública.

En primer lugar, en general, se dan mayores niveles de cooperación en actuaciones relacionadas con la promoción del bienestar social. Por su parte, atendiendo al tipo de actor con el que se colabora, en coherencia con las hipótesis provenientes de la literatura sobre las coaliciones de gobernanza, existe una cierta mayor implicación de actores económicos de la sociedad local (empresas) en actuaciones destinadas al desarrollo económico del municipio, frente a la colaboración con actores cívicos (asociaciones) implicados en mayor medida en todas las áreas de política local y, de forma especial, en aquellas destinadas a la promoción del bienestar social, de la misma forma que para la colaboración con otras unidades dentro del Ayuntamiento. Ello vendría a reforzar la hipótesis planteada al inicio en relación con la existencia de redes de colaboración propias en función del área de políticas públicas: con actores económicos del municipio para promover el desarrollo económico, frente a actores cívicos implicados en políticas destinadas a la promoción del bienestar social. 
TABLA 12. OBJETIVO PERSEGUIDO POR EL MUNICIPIO EN FUNCIÓN DEL TIPO DE GOBERNANZA

\begin{tabular}{|c|c|c|c|c|c|c|c|c|}
\hline & Media Gobernanza & $\begin{array}{l}\text { Gobernanza } \\
\text { Multinivel }\end{array}$ & Empresas & Entidades & $\begin{array}{c}\text { Gobernanza } \\
\text { Externa }\end{array}$ & $\begin{array}{l}\text { Gobernanza } \\
\text { Interna }\end{array}$ & & \\
\hline \multirow{6}{*}{$\begin{array}{l}\text { Promoción } \\
\text { económica }\end{array}$} & \multirow{3}{*}{$\begin{array}{l}\text { Cohesión social y ac- } \\
\text { cesibilidad }\end{array}$} & Media & 22,65 & 12,21 & 14,52 & 31,90 & 22,89 & 31,66 \\
\hline & & $\mathrm{N}$ & 72 & 71 & 70 & 70 & 71 & 69 \\
\hline & & Desv. Típica & 12,98 & 13,60 & 26,15 & 30,39 & 19,96 & 19,57 \\
\hline & \multirow{3}{*}{ Fomento NTIC } & Media & 22,71 & 12,29 & 14,73 & 31,88 & 22,98 & 31,67 \\
\hline & & $\mathrm{N}$ & 71 & 70 & 69 & 69 & 70 & 68 \\
\hline & & Desv. Típica & 13,06 & 13,69 & 26,28 & 30,62 & 20,09 & 19,71 \\
\hline $\begin{array}{l}\text { M e d i a } \\
\text { agregada }\end{array}$ & 12,25 & 14,62 & 31,89 & 22,93 & 31,66 & 22,68 & & \\
\hline \multirow{6}{*}{$\begin{array}{l}\text { Bienestar } \\
\text { social }\end{array}$} & \multirow{3}{*}{$\begin{array}{l}\text { Empleo/nuevos ni- } \\
\text { chos }\end{array}$} & Media & 22,83 & 12,38 & 14,73 & 32,37 & 23,21 & 31,70 \\
\hline & & $\mathrm{N}$ & 71 & 70 & 69 & 69 & 70 & 68 \\
\hline & & Desv. Típica & 12,98 & 13,62 & 26,28 & 30,37 & 19,91 & 19,71 \\
\hline & \multirow{3}{*}{$\begin{array}{l}\text { Oferta Equipamien- } \\
\text { tos y Servicios pú- } \\
\text { blicos }\end{array}$} & Media & 22,82 & 12,14 & 14,49 & 32,37 & 23,10 & 32,03 \\
\hline & & $\mathrm{N}$ & 71 & 70 & 69 & 69 & 70 & 68 \\
\hline & & Desv. Típica & 12,99 & 13,69 & 26,34 & 30,37 & 20,02 & 19,47 \\
\hline $\begin{array}{l}\text { M e d i a } \\
\text { agregada }\end{array}$ & 22,82 & 12,26 & 14,61 & 32,37 & 23,15 & 31,86 & & \\
\hline
\end{tabular}

Fuente: elaboración propia.

Nota: Indicadores medidos en una escala o_100.

\section{CONCLUSIONES}

Este artículo se ha destinado al análisis de actuaciones de regeneración urbana desarrolladas en municipios españoles en el marco de la iniciativa comunitaria URBANA (2007-2013), tanto en relación con el contenido de dichas actuaciones, lo que hemos denominado dimensión sustantiva, como la forma en que estas se gestionan, lo que hemos denominado dimensión procedimental. A su vez, parte del trabajo se ha centrado en el estudio de la relación entre ambas dimensiones, concretamente, el desarrollo de mecanismos de gobernanza local en el marco de diversos tipos de política pública de regeneración urbana.

Ello se ha llevado a cabo a partir del análisis de información recabada en 58 municipios españoles, a través de la administración de un cuestionario a técnicos municipales implicados en el desarrollo de estas iniciativas.

En primer lugar, del análisis de la dimensión procedimental se desprende el establecimiento de mecanismos de coordinación especialmente altos en el ámbito local, ya sea entre áreas municipales o con actores de la sociedad local. Por el contrario, en términos generales, es menor la colaboración entre administraciones, concretamente con otros municipios y Diputación provincial, aumentando cuando se trata de niveles superiores de gobierno (europeo, central o autonómico).

En relación con la dimensión sustantiva, en cuanto a los objetivos perseguidos para el municipio, los análisis han puesto en evidencia que las iniciativas de regeneración urbana que se han puesto en marcha en España en el marco de la iniciativa URBANA (2007-2013) no solamente se circunscriben a la búsqueda de la promoción económica de las ciudades, sino que en ellas se busca además la consecución de otros objetivos, como es la búsqueda de bienestar, bien a través de la mejora de equipamientos y servicios públicos, o bien a través de actuaciones destinadas la integración social de la ciudadanía. Sin embargo, y aunque es un principio planteado en el marco de las iniciativas desarrolladas en el contexto de la iniciativa URBANA (2007-2013), aún no existe una amplia cobertura o carácter integral en cuanto a la diversidad de áreas de política pública implicadas en las mismas, así como el ámbito territorial al que se circunscriben, siendo más bien enfoques fragmentados y parciales, a excepción de una pequeña parte de la muestra, cuyas actuaciones afectarían a la ciudad en su conjunto.

Finalmente, los resultados obtenidos con estos análisis ponen de manifiesto una relación entre la dimensión procedimental y la dimensión sustantiva, mostrando que efectivamente los mecanismos de nueva gobernanza local se dan con niveles de importancia diversos así como el tipo de actor con el que se establece la relación si atendemos al grado de cobertura política y el tipo de objetivo que se persigue para el municipio. Por una parte, a medida que se implica un mayor número de áreas de política local para el desarrollo de la iniciativa de regeneración urbana, se da una mayor colaboración con otros actores. Por su parte, existe una mayor implicación de 
actores económicos de la sociedad local (empresas) en actuaciones destinadas al desarrollo económico del municipio, frente a la colaboración con actores cívicos (asociaciones) implicados en mayor medida en todas las áreas de política local y, de forma especial, en aquellas destinadas a la promoción del bienestar social.

En este sentido, será necesario indagar sobre esta relación en futuros trabajos, destinados en su caso a la comparación entre el caso español y otros, al objeto de conocer en qué medida dicha relación se da en otros contextos nacionales e institucionales. Así como, dentro del caso español, otras iniciativas de regeneración urbana desarrolladas no necesariamente en el marco de las iniciativas europeas del programa URBANA, pues podría darse un cierto sesgo en los resultados. Dadas las características propias del programa URBANA, éste podría inducir a la conformación de unas características específicas de las actuaciones, tanto en su cobertura como en el desarrollo de mecanismos de gobernanza, ya que ambos aspectos se encuentran muy presentes en el espíritu y conformación de dicho programa.

\section{BIBLIOGRAFÍA}

BIANCHINI, F. y PARKINSON, M. eds. (1993), Cultural policy and urban regeneration: the West European Experience. Manchester: Manchester University Press.

BLAIR, P. (1991), “Trends in local autonomy and democracy”, en Local Government in Europe. Trends and developments. London: MacMillan.

BLANCO, I. (2009), “Gobernanza urbana y políticas de regeneración: el caso de Barcelona”, Revista Española de Ciencia Política. 20:125-146.

BLANCO, I.; BONET, J.; y WALLISER, A. (2011): “Urban governance and regeneration policies in historic city centres: Madrid and Barcelona". Urban research and practice. Vol. 4. No 3. November 2011, 326-343.

BORJA, J. (2003), La ciudad conquistada. Madrid: Alianza.

BORJA, J. (1998), “Ciudadanía y espacio público”. Ambiente y desarrollo XIV: 13-22.

BRENDAN, B. y SHINE, K.T. (2001), “Regeneración urbana en Dublín”. Ciudad y Territorio: Estudios Territoriales 129: 461-474.

BURSTEIN, P. (1991), “Policy domains: organization, culture and policy outcomes". Annual Review of Sociology 17: 327-50. DOI: 10.1146/annurev.so.17.080191.001551.

CASTELLS, M. (1986), El desafío tecnológico. Madrid: Alianza.

COMISIÓN EUROPEA:

- (2000): Comunicación de la Comisión a los Estados miembros por la que se fijan las orientaciones de una Iniciativa comunitaria relativa a la regeneración económica y social de las ciudades y de los barrios en crisis con el fin de fomentar un desarrollo urbano sostenible - URBAN II (DOCE C 141/04, 19.05.2000)

- (2006), Estrategia temática para el medio ambiente urbano. COM (2005) 718 final. Bruselas: Comisión Europea.

- (2006): Política de Cohesión y ciudades: la contribución urbana al crecimiento y el empleo en las regiones. COM (2006) 385 final. Bruselas: Comisión Europea.

CONSEJO DE EUROPA. (2009), “Manifesto for a new urbanity”. European Urban Charter II. Estrasburgo.

DAVIES, J. (2002), “The governance of Urban Regeneration. A critique of the 'governing without government' thesis". Public Administration 2:301-322.

DAVoudi, S.; Evans, N.; Governa, F.; SANTANGElo, M. (2008), “Territorial governance in the making approaches, methodologies, practices". Boletín de la AGE. N 46: 33-52.

DE GREGORIO HURTADO, S. (2010): “El desarrollo de las iniciativas comunitarias URBAN y URBAN II en las periferias degradadas de las ciudades españolas. Una contribución a la práctica de la regeneración urbana en España”. Ciudades 13: 39-59.

FLORIDA, R. (2009): Las Ciudades creativas. Barcelona: Paidós.

GonZÁLEZ MEDINA, M. (2011), “Elementos de una política urbana europea. Experiencias de gestión del desarrollo urbano en Alemania y España”. Revista Española de Ciencia Política 27:45-67.

HOOGHE, L. y MARKS, G.N. (2001), "Types of Multi-Level Governance”. European Integration online Papers 5: 1-24.

INSTITUTO UNIVERSITARIO DE URBANÍSTICA DE LA UNIVERSIDAD DE VALLADOLID (2010): Regeneración urbana in- 
tegrada en Europa. Documento de síntesis, disponible en: http://www.fomento.gob.es/NR/rdonlyres/B8F44D9E47ED-44DE-8FEC-47FFE2FC630B/111525/3_survey_on_integrated.pdf

JONES, P. y EVANS, J. (2006), "Urban Regeneration, Governance and the State: Exploring Notions of Distance and Proximity". Urban Studies 9:1491-1509. DOI: 10.1080/00420980600749951.

KEARS, A. y TUROK, I. (2000), "Power, responsibility and governance in Britains new urban policy". Journal of Urban Affairs 22: 175-191. DOI: 10.1111/0735-2166.00049.

KNOKE, D. (1990), Political Networks: The Structural Perspective. New York: Cambridge University Press. DOI: 10.1017/CBO9780511527548.

LOGAN, J. y MOLOTCH, H. (1987), Urban fortunes. Berkeley: University of California Press.

LOWI, T. (1964), “American business, public policy, case studies and political theory”. World Politics 16: 677-693. DOI: $10.2307 / 2009452$.

MARSHALL, A.J. (2004), "Europeanization at the urban level: local actors, institutions and the dynamics of multi-level interaction". Journal of European Public Policy. 12: 668-686. DOI: 10.1080/13501760500160292.

NEL·LO, O. (2010): “The challenges of urban renewal. Ten lessons from catalan experience", en Análise Social. Vol. XLV (197): 685-715.

Ostrom, E. (1986), "An agenda for the study of institutions". Public Choice 1:3-25.

PADDISON, R. (1993), "City marketing. Image reconstruction and Urban Regeneration". Urban Studies Review 30: 339-350. DOI: 10.1080/00420989320080331.

PAINTER, J. y GOODWIN, M. (1995), "Local governance and concrete research: investigating the uneven development of regulation”. Economy and society, 24 (3): 334-356. DOI: 10.1080/03085149500000014.

PARÉS, M.; Bonet-MARTí, J.; MARTí-COSTA, M. (2011), "Does participation really matter in Urban Regeneration Policies?" Exploring governance networks in Catalonia". Urban Affairs Review 10: 1-34.

PierRE, J. (1999), "Models of Urban Governance. The Institutional Dimension of Urban Politics". Urban Affairs Review 34: 372-396. DOI: 10.1177/10780879922183988.

RHODES, R. (1997), Understanding Governance: Policy Networks, Governance, Reflexivity and Accountability. Buckingham: Open University Press.

ROBERTS, P. y SYKES, H. (eds.) (2000), Urban Regeneration. A handbook. London: Sage Publications.

SASSEN, S. (2009), Territorio, autoridad y derechos. Madrid: Akal.

SCHARPF, F.W. (1997), Games Real Actors Play: Actor-centered institutionalism in Policy research. Boulder, CO: Westview Press.

StONE, C.N. (1989), Regime politics. Lawrence: University of Kansas Press.

ZAMORA, E y MERINERO, R. (2012), "Desarrollo urbano integral: orientaciones metodológicas para el diseño de políticas públicas en barrios”. Ciudad y Territorio, n ${ }^{173}$, pp. 445-462. 